\title{
Fourier spectrum of curvilinear gratings of the second order
}

\author{
Isaac Amidror \\ Laboratoire de Systèmes Périphériques, Ecole Polytechnique Fédérale de Lausanne, 1015 Lausanne, Switzerland
}

Received July 24, 1997; revised manuscript received October 7, 1997; accepted October 20, 1997

\begin{abstract}
The class of second-order curvilinear gratings consists of all the curvilinear gratings that are obtained by second-order spatial transformations of periodic gratings. It includes, for example, circular, elliptic, and hyperbolic gratings as well as circular, elliptic, and hyperbolic zone plates. Such structures occur quite frequently in optics, and their Fourier transforms may arise, for instance, in connection with the Fraunhofer diffraction patterns generated by these structures. I present the two-dimensional Fourier spectra of the most important second-order curvilinear gratings for gratings having any desired intensity profile (cosinusoidal, sawtooth wave, square wave, etc.). These analytic results are also illustrated by figures showing the various gratings and their spectra as they are obtained on a computer by two-dimensional fast Fourier transform. (C) 1998 Optical Society of America [S0740-3232(98)00304-4]

OCIS codes: $050.2770,070.2580$.
\end{abstract}

\section{INTRODUCTION}

In a previous paper ${ }^{1} \mathrm{I}$ investigated the structure and the properties of the Fourier spectrum of radially periodic functions, i.e., circularly symmetric images on the twodimensional (2D) plane whose intensity profile along the radius is periodic. Such functions are obtained by applying a polar-to-Cartesian coordinate transformation on an original onefold periodic function (grating) $p(r)$, namely, by replacing $r$ by $\sqrt{x^{2}+y^{2}}: \quad r(x, y)=p\left(\sqrt{x^{2}+y^{2}}\right)$. However, radially periodic images are in fact only one particular case of a larger class of functions that consists of all the second-order spatial transformations of periodic gratings. This class includes, for example, parabolic, elliptic, and hyperbolic gratings; circular, elliptic, and hyperbolic zone gratings (zone plates), etc. Like radially periodic images, such functions occur quite frequently in optics, ${ }^{2-4}$ and their Fourier transforms may arise, for example, in connection with the Fraunhofer diffraction patterns generated by these structures. Other applications in optics include gratings in various optoelectronic applications ${ }^{5}$ and in moiré-related applications. The full understanding of the structure and the properties of the Fourier spectra of such functions may be very useful in such cases.

In order to find analytically the Fourier spectrum of a second-order curvilinear grating with any given intensity profile (square wave, sawtooth wave, etc.) we need to know first the Fourier spectra of the corresponding curvilinear gratings with a cosinusoidal or a sinusoidal intensity profile. Therefore, after presenting the basic notions about curvilinear gratings (Section 2) and about secondorder curvilinear gratings in particular (Section 3), we start by finding the Fourier spectra of the main families of second-order curvilinear gratings with a cosinusoidal or sinusoidal intensity profile (Sections 4-7). Then in Section 8 we generalize these results for gratings with any desired intensity profile, using the Fourier decomposition of their intensity profile.

Note that the Fourier transform conventions throughout this paper have been adapted to Bracewell's notations ${ }^{6}$; thus the Fourier transform of a function $f(x, y)$ is given by

$$
F(u, \nu)=\int_{-\infty}^{\infty} \int_{-\infty}^{\infty} f(x, y) \exp [-i 2 \pi(u x+\nu y)] \mathrm{d} x \mathrm{~d} y .
$$

\section{CURVILINEAR GRATINGS}

A curvilinear grating can be defined as the repetitive structure that is obtained on the $x, y$ plane by the application of an appropriate nonlinear spatial transformation on an initial uncurved, periodic grating. One may think of this transformation as an operation that "bends," or nonlinearly stretches, the original periodic grating according to a given mathematical rule.

Let $r(x, y)$ (denoting the reflectance or the transmittance at location $x, y$ ) be the curvilinear grating that is obtained by bending the $2 \mathrm{D}$ onefold periodic grating $p\left(x^{\prime}\right)$, i.e., by replacing $x^{\prime}$ with the function $g_{1}(x, y)$ : $r(x, y)=p\left[g_{1}(x, y)\right]$ (see various examples in Fig. 1 ). The intensity profile of the original, uncurved periodic grating, $p\left(x^{\prime}\right)$, or sometimes its one-dimensional (1D) section along the $x^{\prime}$ axis, is called the periodic profile of the curvilinear grating $r(x, y)$. The periodic profile of a curvilinear grating may be cosinusoidal, a square wave, a sawtooth wave, or any other periodic waveform. The function $x^{\prime}=g_{1}(x, y)$ that bends $p\left(x^{\prime}\right)$ into the curvilinear grating $r(x, y)$ is called the bending transformation.

A curvilinear grating $r(x, y)=p\left[g_{1}(x, y)\right]$ is therefore characterized by two basic and independent properties: (a) its geometric layout in the $x, y$ plane, i.e., the locus of the centers of its curvilinear corrugations in the $x, y$ plane, which is defined by the bending transforma- 
tion $x^{\prime}=g_{1}(x, y)$, and (b) the intensity behavior across each of the curvilinear corrugations, which is determined by the periodic profile $p\left(x^{\prime}\right)$.

Example. Assume that we are given a 2D cosinusoidal grating $p\left(x^{\prime}\right)=\cos \left(2 \pi f x^{\prime}\right)$ over the $x, y$ plane [Fig. 1(a)] and that we bend its parallel straight corrugations into parallel parabolas of the shape $y=a x^{2}+c$, without changing their cosinusoidal profile form [see Fig. 4(a) below, in Appendix A]. This can be described mathematically as a nonlinear transformation $x^{\prime}=g_{1}(x, y)$ $=y-a x^{2}$, where $a$ is a nonzero constant that defines the bending rate of the parabolas. ${ }^{7}$ (Notice that the level lines $x^{\prime}=n$ of the surface $x^{\prime}=y-a x^{2}$ over the $x, y$ plane are indeed the required parabolas $y=a x^{2}+n$.) The parabolic cosinusoidal grating obtained by applying this bending transformation on the original grating $p\left(x^{\prime}\right)$ is given, therefore, by $r(x, y)=p(y$ $\left.-a x^{2}\right)=\cos \left[2 \pi f\left(y-a x^{2}\right)\right]$. Its geometric layout is given by the locus of its maxima in the $x, y$ plane, namely, $2 \pi f\left(y-a x^{2}\right)=2 \pi k, k \in \mathbb{Z}$, and its periodic profile is $\cos \left(2 \pi f x^{\prime}\right)$.

Now, assuming that the spectrum of the original periodic grating $p\left(x^{\prime}\right)$ is known, how does the application of the transformation (coordinate change) $x^{\prime}=g_{1}(x, y)$ on this grating affect the spectral domain? Unfortunately, if the coordinate change $g_{1}(x, y)$ in the image domain is nonlinear, no general rule exists that tells how the spectrum will be influenced, and the spectra of $p\left[g_{1}(x, y)\right]$ must be individually found for each transformation $g_{1}(x, y)$ on a case-by-case basis. It is therefore the aim of this paper to present the Fourier spectra of the most important second-order curvilinear gratings and thus to open the way to a full understanding of the properties of such gratings and of their spectra.

\section{SECOND-ORDER CURVILINEAR GRATINGS}

As illustrated in the example above, the bending transformation $z=g(x, y)$ (using here $z$ rather than $x^{\prime}$ ) can be also considered a $2 \mathrm{D}$ curved surface in $\mathbb{R}^{3}$ whose level lines $z=n(n \in \mathbb{Z})$, or their projection on the $x, y$ plane, determine the geometric layout of the curvilinear grating. We will call this surface the generating surface of the curvilinear grating. Note that the generating surface need not necessarily be defined by an explicit function $z$ $=g(x, y)$, as in the examples above, and in the general case it can be given implicitly in the form $G(x, y, z)$ $=0$.

A curvilinear grating whose generating surface is a surface of the second order $^{8}$ will be called a second-order curvilinear grating. In other words, a second-order curvilinear grating is a curvilinear grating whose bending transformation is given by an equation $G(x, y, z)=0$ that is a polynomial of the second degree in $x, y, z$. In the most general case this equation has the form

$$
\begin{aligned}
a_{11} x^{2}+a_{22} y^{2}+a_{33} z^{2}+2 a_{12} x y+2 a_{13} x z+2 a_{23} y z \\
+2 a_{14} x+2 a_{24} y+a_{34} z+a_{44}=0 .
\end{aligned}
$$

As is well known in three-dimensional (3D) analytic geometry, second-order surfaces can be classified into 17 dif- ferent types depending on the coefficients in this general equation. In other words, one may transform this general equation by parallel translations and rotations in $\mathrm{R}^{3}$ into one of 17 canonical forms, each of which corresponds to a certain class of surfaces. The full list of these 17 surfaces is given, for example, in Refs. 8 and 9 . However, since five of these surfaces are purely imaginary and three others are degenerate cases consisting of planes, we are left, in fact, with only nine different types of interesting second-order surfaces: an ellipsoid (including a sphere), a one-sheet hyperboloid, a two-sheet hyperboloid, an elliptic paraboloid, a hyperbolic paraboloid ("saddle"), a conic surface, an elliptic cylinder, a hyperbolic cylinder, and a parabolic cylinder (the last three surfaces have one constant dimension).

It should be noted, however, that although in analytic geometry all the different $3 \mathrm{D}$ rotations and translations of a given surface within $\mathbb{R}^{3}$ result in equivalent surfaces, the curvilinear gratings these surfaces generate by their level lines may be completely different. For instance, different orientations of a conic surface within $\mathbb{R}^{3}$ may give rise to circular, elliptic, parabolic, or hyperbolic curvilinear gratings, depending on the inclination angle of the conic surface.

In fact, from the point of view of the Fourier transform, only the application of affine transformations (such as rotations and translations) in the $x$ and $y$ coordinates will give equivalent gratings, while the application on the surface of any nonaffine transformation in $x$ and $y$ or any transformation involving the $z$ coordinate may result in substantial changes in the generated gratings and in their spectra. Even a simple translation of the surface along the $z$ axis will, in general, influence the relative phase of the curvilinear grating and its spectrum in a nontrivial way (see Appendix B).

We see, therefore, that the nine different types of second-order surfaces may give rise to many different types of second-order curvilinear gratings. Although the geometric layout of these gratings may consist only of curves of the second order (circles, ellipses, parabolas, hyperbolas, and possibly straight lines), these layouts may still differ in parameters such as the rules governing the curve spacing [cf. circular grating versus circular zone grating in Figs. 1(i) and 1(b), respectively; as we will see below, their spectra are indeed substantially different].

However, many of these different second-order curvilinear gratings are of limited practical interest; such are, for example, the highly oscillatory grating $\cos [2 \pi f(1 / x)]$ or the grating $\cos \left(2 \pi f \sqrt{y-x^{2}}\right)$, which is defined only inside the parabola $y=x^{2}$. Therefore we will concentrate here only on classes of second-order curvilinear gratings that present a certain theoretical interest or that are most likely to occur in optics and in applications. We start in Sections 4-7 with gratings that have a cosinusoidal or a sinusoidal periodic profile, and only then, in Section 8 , will we generalize these results to gratings with any desired periodic profile. We start in Section 4 with gratings obtained from parabolic surfaces, which include the various forms of zone gratings. Then, in Sections 5, 6 and 7 we proceed to gratings obtained, respectively, from elliptic, conic, and hyperbolic surfaces. Each case is illustrated by a figure of one representative grating, and its 
Fourier spectrum is illustrated and validated by $2 \mathrm{D}$ fast Fourier transform. (Note that FFT artifacts that occur in some of the spectra are explained in the figure legends.)

\section{GRATINGS OBTAINED FROM PARABOLIC SURFACES, AND THEIR SPECTRA}

In this section I discuss gratings that are obtained from parabolic surfaces: the elliptic paraboloid, the hyperbolic paraboloid ("saddle"), and the parabolic cylinder. In fact, the gratings obtained from parabolic surfaces have a special significance in optics, since they constitute the important grating family known as zone gratings or zone plates, which are often used as focusing devices based on diffraction. ${ }^{2,3,10,11}$

\section{A. Circular Zone Grating}

The cosine circular zone grating [Fig. 1(b)] is defined by

$$
r_{1}(x, y)=\cos \left[2 \pi f\left(x^{2}+y^{2}\right)\right] .
$$

Its generating surface is the top-opened circular paraboloid $z=x^{2}+y^{2}$.
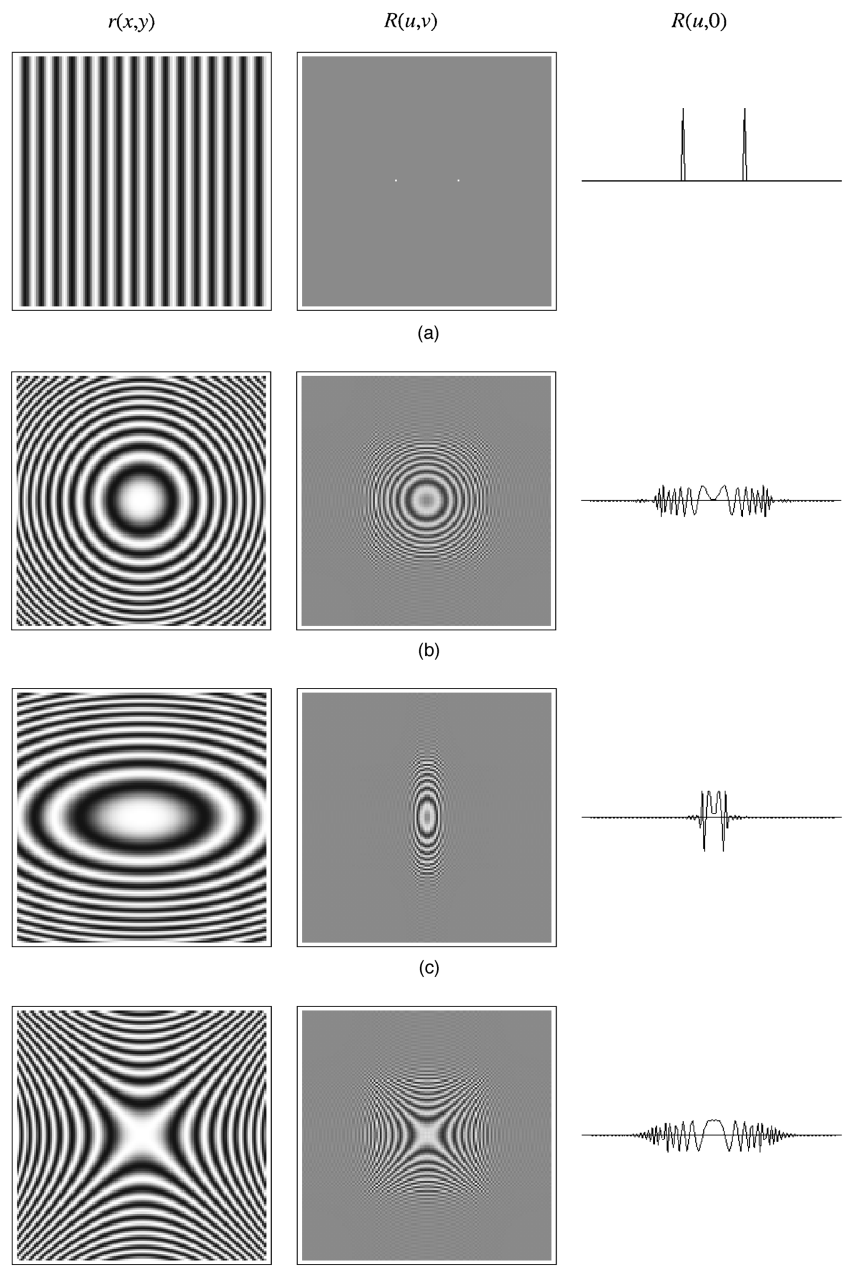

(d)
Let us find the spectrum $R_{1}(u, v)$ of this function. According to the trigonometric identity $\cos (\alpha+\beta)$ $=\cos \alpha \cos \beta-\sin \alpha \sin \beta$, we have

$$
\begin{aligned}
r_{1}(x, y)= & \cos \left(2 \pi f x^{2}\right) \cos \left(2 \pi f y^{2}\right) \\
& -\sin \left(2 \pi f x^{2}\right) \sin \left(2 \pi f y^{2}\right) .
\end{aligned}
$$

Consider first the 1D function $r_{c}(x)=\cos \left(2 \pi f x^{2}\right)$. The 1D spectrum of this function is (see Fig. 2):

$$
\begin{aligned}
R_{c}(u) & =\frac{1}{2 \sqrt{f}}\left[\cos \left(\frac{\pi}{2 f} u^{2}\right)+\sin \left(\frac{\pi}{2 f} u^{2}\right)\right] \\
& =\frac{1}{\sqrt{2 f}} \cos \left(\frac{\pi}{2 f} u^{2}-\frac{\pi}{4}\right)
\end{aligned}
$$

[adapted from Eq. (7) in Ref. 12]. Thanks to the separable-product theorem ${ }^{13}$ we obtain
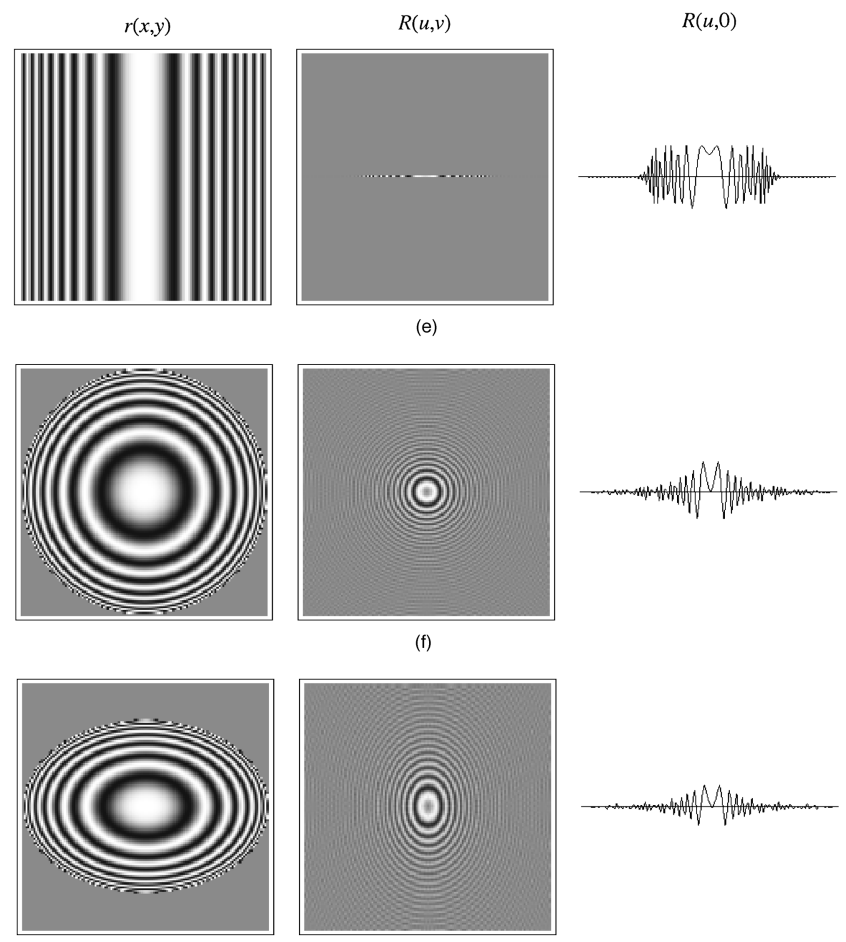

(g)
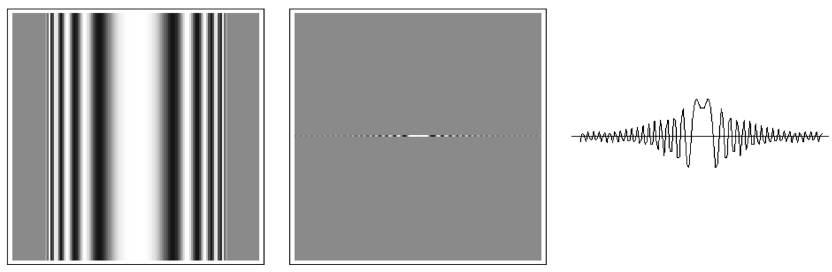

Fig. 1. Continues on facing page. 
$\cos \left(2 \pi f a x^{2}\right) \cos \left(2 \pi f b y^{2}\right)$

$$
\begin{aligned}
\leftrightarrow & \frac{1}{2 \sqrt{f}}\left[\cos \left(\frac{\pi}{2 f} u^{2}\right)+\sin \left(\frac{\pi}{2 f} u^{2}\right)\right] \\
& \times \frac{1}{2 \sqrt{f}}\left[\cos \left(\frac{\pi}{2 f} v^{2}\right)+\sin \left(\frac{\pi}{2 f} v^{2}\right)\right],
\end{aligned}
$$

which gives, after some trigonometric simplifications,

$$
=\frac{1}{4 f}\left[\cos \frac{\pi}{2 f}\left(u^{2}-v^{2}\right)+\sin \frac{\pi}{2 f}\left(u^{2}+v^{2}\right)\right] .
$$

Similarly, thanks to the separable-product theorem we have
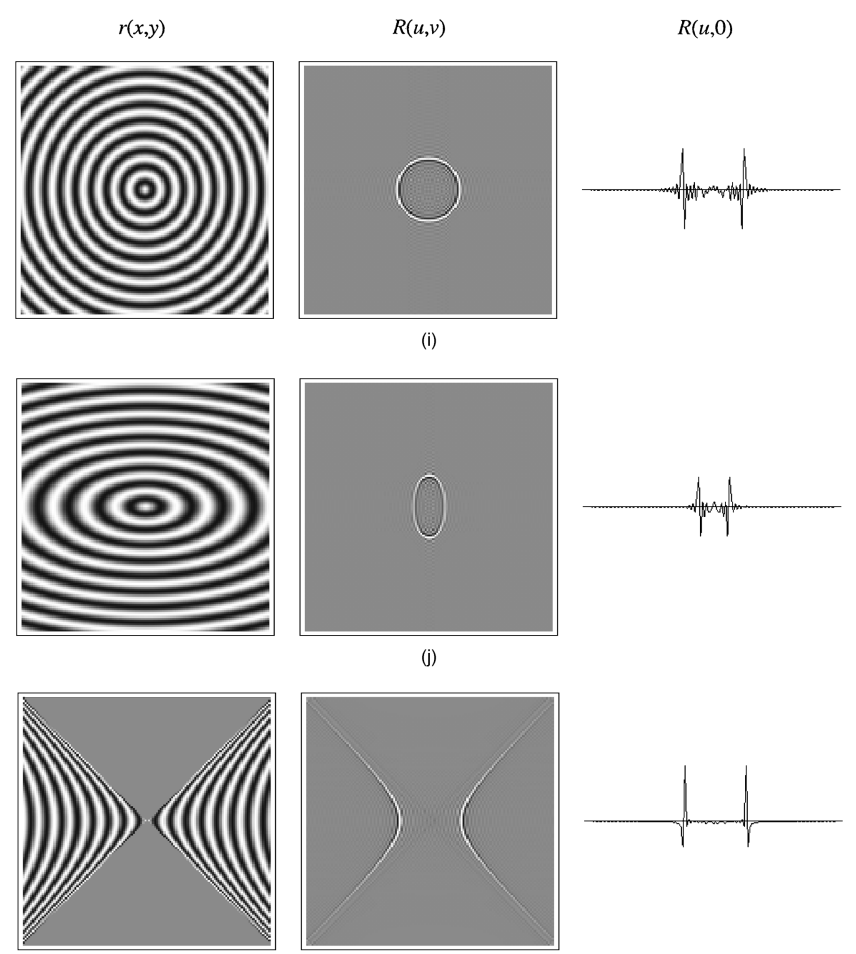

(k)
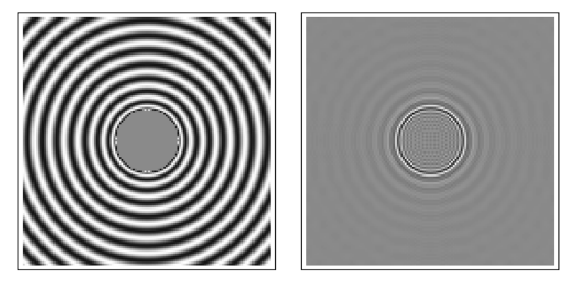

(I) $\sin \left(2 \pi f a x^{2}\right) \sin \left(2 \pi f b y^{2}\right)$

$$
\leftrightarrow \frac{1}{4 f}\left[\cos \frac{\pi}{2 f}\left(u^{2}-v^{2}\right)-\sin \frac{\pi}{2 f}\left(u^{2}+v^{2}\right)\right]
$$

and therefore we finally obtain

$$
R_{1}(u, v)=\frac{1}{2 f} \sin \left[\frac{\pi}{2 f}\left(u^{2}+v^{2}\right)\right]
$$

Similarly, using the spectrum of the 1D function $r_{s}(x)$ $=\sin \left(2 \pi f x^{2}\right)$ (see Fig. 2),

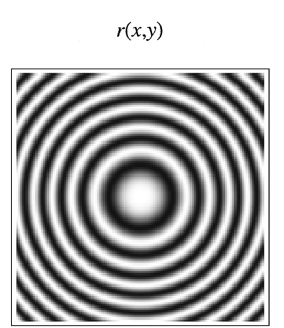

$R(u, v)$

$R(u, 0)$

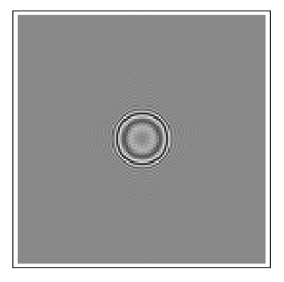

(m)
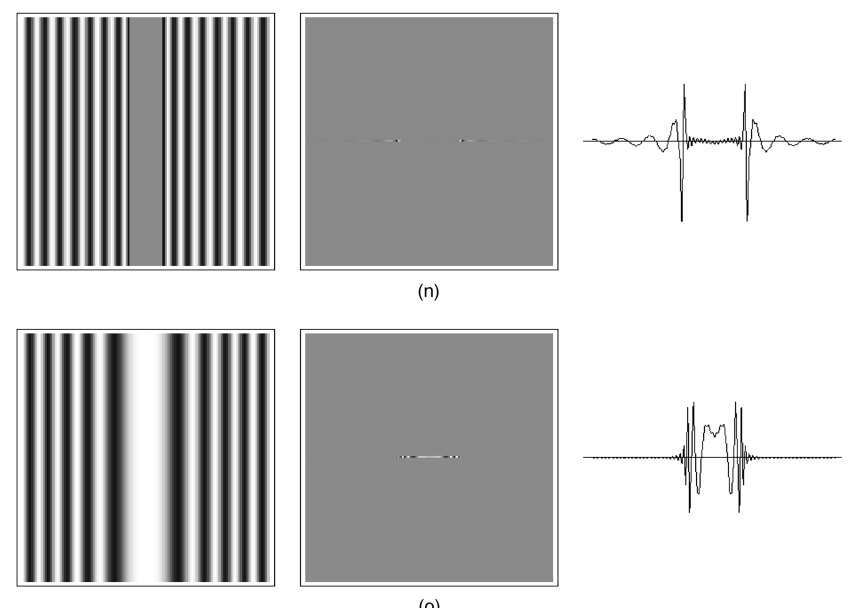

Fig. 1. Various curvilinear gratings $r(x, y)$ with a periodic-profile waveform of $\cos (2 \pi f x)$ (with $f=1$ ), and their spectra $R(u, v)$ as obtained on computer by 2D discrete Fourier transform (DFT): (a) straight grating, $\cos (2 \pi f x)$; (b) circular zone grating, $\cos \left[2 \pi f\left(x^{2}\right.\right.$ $\left.\left.+y^{2}\right) / 8\right]$; (c) elliptic zone grating, $\cos \left[2 \pi f\left(\frac{1}{4} x^{2}+y^{2}\right) / 8\right]$; (d) hyperbolic zone grating, $\cos \left[2 \pi f\left(x^{2}-y^{2}\right) / 8\right]$; (e) linear zone grating, $\cos \left(2 \pi f x^{2} / 8\right)$; ( f) sphere projection grating, $\cos \left(2 \pi f \sqrt{64-x^{2}-y^{2}}\right)$; (g) ellipsoid projection grating, $\cos \left(2 \pi f \sqrt{64-x^{2}-2 y^{2}}\right)$; (h) cylinder projection grating, $\cos \left(2 \pi f \sqrt{36-x^{2}}\right)$; (i) circular grating, $\cos \left(2 \pi f \sqrt{x^{2}+y^{2}}\right)$; (j) elliptic grating, $\cos \left(2 \pi f \sqrt{\frac{1}{4} x^{2}+y^{2}}\right)$; (k) hyperbolic grating, $\cos \left(2 \pi f \sqrt{x^{2}-y^{2}}\right)$; (l) one-sheet hyperboloid projection grating, $\cos \left(2 \pi f \sqrt{x^{2}+y^{2}-4}\right)$; (m) two-sheet hyperboloid projection grating, $\cos \left(2 \pi f \sqrt{x^{2}+y^{2}+16}\right)$; (n) laterally opened hyperbolic cylinder projection grating, $\cos \left(2 \pi f \sqrt{x^{2}-1}\right)$; (o) top-opened hyperbolic cylinder projection grating, $\cos \left(2 \pi f \sqrt{x^{2}+16}\right)$. Since all these cases are centrosymmetric, their spectra have no imaginary parts. The righthand figure in each row shows a cross section through the horizontal $u$ axis of the spectrum. Note that the oscillations in the spectra of the zone gratings (b)-(e) fade out short of the spectrum border since the DFT cannot find higher frequencies in the corresponding finite-sized, sampled functions in the image domain; in reality these spectra oscillate ad infinitum without fading out. Note also the DFT rippling artifacts in some of the spectra. 

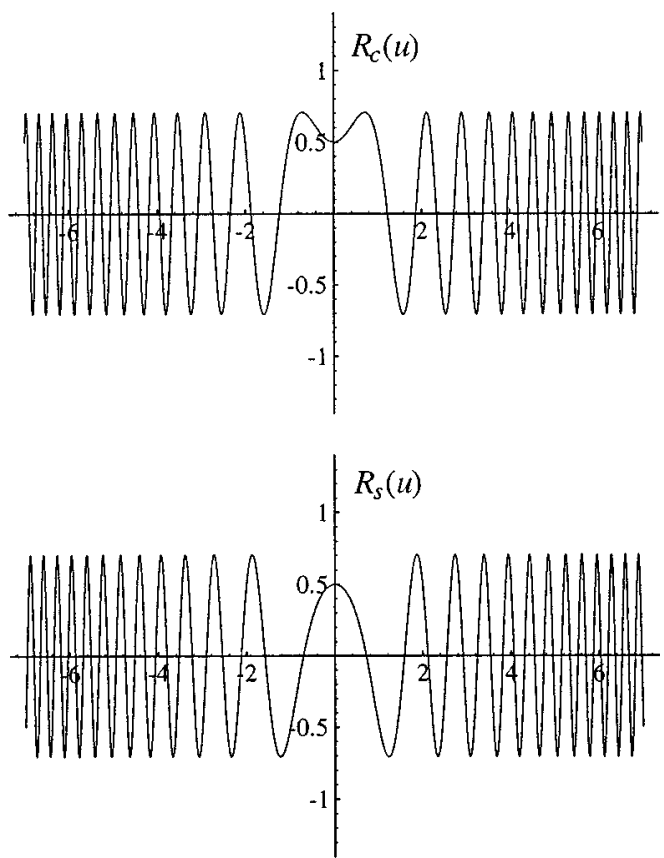

Fig. 2. $R_{c}(u)$ and $R_{s}(u)$, the 1D Fourier transforms of the functions $r_{c}(x)=\cos \left(2 \pi f x^{2}\right)$ and $r_{s}(x)=\sin \left(2 \pi f x^{2}\right)($ with $f=1)$.

$$
\begin{aligned}
R_{s}(u) & =\frac{1}{2 \sqrt{f}}\left[\cos \left(\frac{\pi}{2 f} u^{2}\right)-\sin \left(\frac{\pi}{2 f} u^{2}\right)\right] \\
& =\frac{1}{\sqrt{2 f}} \sin \left(\frac{\pi}{2 f} u^{2}+\frac{\pi}{4}\right)
\end{aligned}
$$

[adapted from Eq. (1) in Ref. 14], we find that the sinusoidal counterpart of $r_{1}(x, y), \quad r_{2}(x, y)=\sin \left[2 \pi f\left(x^{2}\right.\right.$ $\left.+y^{2}\right)$, has the Fourier spectrum $R_{2}(u, v)$ $=\frac{1}{2 f} \cos \left[\frac{\pi}{2 f}\left(u^{2}+v^{2}\right)\right]$.

\section{B. Elliptic Zone Grating}

The elliptic zone grating [Fig. 1(c)], a generalization of the circular zone grating, is obtained by scaling in $x$ and $y$ :

$$
r_{1}(x, y)=\cos \left\{2 \pi f\left[(b x)^{2}+(c y)^{2}\right]\right\} .
$$

Therefore its generating surface is the top-opened elliptic paraboloid $z=(b x)^{2}+(c y)^{2}$, and its spectrum is given, according to the $2 \mathrm{D}$ similarity theorem, ${ }^{15}$ by

$$
R_{1}(u, v)=\frac{1}{2 f|b c|} \sin \left\{\frac{\pi}{2 f}\left[\left(\frac{u}{b}\right)^{2}+\left(\frac{v}{c}\right)^{2}\right]\right\} .
$$

Similarly, the spectrum of the sinusoidal counterpart of $r_{1}(x, y), \quad r_{2}(x, y)=\sin \left\{2 \pi f\left[(b x)^{2}+(c y)^{2}\right]\right\}$, is given by $R_{2}(u, v)=\frac{1}{2 f}|b c| \cos \left\{\frac{\pi}{2 f}\left[\left(\frac{u}{b}\right)^{2}+\left(\frac{v}{c}\right)^{2}\right]\right\}$.

\section{Hyperbolic Zone Grating}

The hyperbolic zone grating ${ }^{16}$ [Fig. 1(d)], another generalization of the circular zone grating, is given by

$$
r_{1}(x, y)=\cos \left\{2 \pi f\left[(b x)^{2}-(c y)^{2}\right]\right\}
$$

Its generating surface is the hyperbolic paraboloid $z$ $=(b x)^{2}-(c y)^{2}$, and its spectrum is

$$
R_{1}(u, v)=\frac{1}{2 f|b c|} \cos \left\{\frac{\pi}{2 f}\left[\left(\frac{u}{b}\right)^{2}-\left(\frac{v}{c}\right)^{2}\right]\right\} .
$$

Similarly, for the sinusoidal counterpart of $r_{1}(x, y)$, $r_{2}(x, y)=\sin \left\{2 \pi f\left[(b x)^{2}-(c y)^{2}\right]\right\}$, we obtain $R_{2}(u, v)$ $=\frac{1}{2 f}|b c| \sin \left\{\frac{\pi}{2 f}\left[\left(\frac{u}{b}\right)^{2}-\left(\frac{v}{c}\right)^{2}\right]\right\}$.

\section{Linear Zone Grating}

The 2D functions $r_{1}(x, y)=\cos \left(2 \pi f a x^{2}\right)$ and $r_{3}(x, y)$ $=\cos \left(2 \pi f a y^{2}\right)$ are called by analogy linear cosine zone gratings [see Fig. 1(e)]. Their generating surfaces are the parabolic cylinders $z=a x^{2}$ and $z=a y^{2}$, which are constant in the $y$ or in the $x$ direction, respectively.

To find the $2 \mathrm{D}$ spectra of these gratings, we first consider their 1D counterpart, $r_{c}(x)=\cos \left(2 \pi f a x^{2}\right)$, whose spectrum $R_{c}(u)$ has been given in Eq. (1). The 2D spectrum of the linear zone grating $r_{1}(x, y)$ is, therefore, the continuous horizontal line impulse: $R_{1}(u, v)$ $=R_{c}(u) \delta(v)$, and the spectrum of the linear zone grating $r_{3}(x, y)$ is the continuous vertical line impulse: $R_{3}(u, v)=R_{c}(v) \delta(u)$.

As for the sinusoidal counterparts, $r_{2}(x, y)$ $=\sin \left(2 \pi f a x^{2}\right)$ and $r_{4}(x, y)=\sin \left(2 \pi f a y^{2}\right)$, their respective spectra are the horizontal and the vertical line impulses $\quad R_{2}(u, v)=R_{s}(u) \delta(v) \quad$ and $\quad R_{4}(u, v)$ $=R_{s}(v) \delta(u)$, respectively, where $R_{s}(u)$ is the $1 \mathrm{D}$ spectrum of $\sin \left(2 \pi f a x^{2}\right)$ given in Eq. (2).

\section{GRATINGS OBTAINED FROM ELLIPTIC SURFACES, AND THEIR SPECTRA}

This section includes gratings that are obtained from the sphere, the ellipsoid, and the elliptic cylinder.

\section{A. Sphere Projection Grating}

The sine and cosine sphere projection gratings [Fig. 1(f)] are given by

$$
\begin{aligned}
& r_{1}(x, y)=\left\{\begin{array}{ll}
\sin \left(2 \pi f \sqrt{a^{2}-r^{2}}\right) & 0 \leqslant r<a \\
0 & a \leqslant r<\infty
\end{array},\right. \\
& r_{2}(x, y)=\left\{\begin{array}{ll}
\cos \left(2 \pi f \sqrt{a^{2}-r^{2}}\right) & 0 \leqslant r<a \\
0 & a \leqslant r<\infty
\end{array},\right.
\end{aligned}
$$

where $r^{2}=x^{2}+y^{2}$; their generating surface is the sphere of radius $a, z=\sqrt{a^{2}-r^{2}}$.

The spectrum $R_{1}(u, v)$ of $r_{1}(x, y)$ is given by

$$
R_{1}(u, v)=\pi f a^{3 / 2} \frac{1}{\left(f^{2}+q^{2}\right)^{3 / 4}} J_{3 / 2}\left(2 \pi a \sqrt{f^{2}+q^{2}}\right),
$$

where $q^{2}=u^{2}+v^{2}$ and $J_{p}(x)$ is the Bessel function of the first kind of order $p$. We obtain this result from Eq. 2.57 in Ref. 17 by adapting it to our conventions and using the known relation ${ }^{18} J_{3 / 2}(x)=\sqrt{2 / \pi x}(\sin x / x-\cos x)$. Similarly, the spectrum of the cosinusoidal counterpart, $r_{2}(x, y)$, is given by

$$
R_{2}(u, v)=-\pi f a^{3 / 2} \frac{1}{\left(f^{2}+q^{2}\right)^{3 / 4}} Y_{3 / 2}\left(2 \pi a \sqrt{f^{2}+q^{2}}\right),
$$

where $Y_{p}(x)$ is the Bessel function of the second kind of order $p$. 


\section{B. Ellipsoid Projection Grating}

The sine and cosine ellipsoid projection gratings [Fig. $1(\mathrm{~g})$ ] are generalizations of the sphere projection gratings that are obtained by scaling in $x$ and in $y: r_{3}(x, y)$ $=r_{1}(b x, c y), r_{4}(x, y)=r_{2}(b x, c y)$. They are given by the same equations as $r_{1}(x, y)$ and $r_{2}(x, y)$ above where $r^{2}=(b x)^{2}+(c y)^{2}$, and their generating surface is the ellipsoid $z=\sqrt{a^{2}-r^{2}}$.

Therefore by the $2 \mathrm{D}$ similarity theorem ${ }^{15}$ we obtain

$$
\begin{aligned}
R_{3}(u, v) & =\frac{1}{|b c|} R_{1}\left(\frac{u}{b}, \frac{v}{c}\right) \\
& =\frac{1}{|b c|} \pi f a^{3 / 2} \frac{1}{\left(f^{2}+q^{2}\right)^{3 / 4}} J_{3 / 2}\left(2 \pi a \sqrt{f^{2}+q^{2}}\right), \\
R_{4}(u, v) & =\frac{1}{|b c|} R_{2}\left(\frac{u}{b}, \frac{v}{c}\right) \\
& =-\frac{1}{|b c|} \pi f a^{3 / 2} \frac{1}{\left(f^{2}+q^{2}\right)^{3 / 4}} Y_{3 / 2}\left(2 \pi a \sqrt{f^{2}+q^{2}}\right),
\end{aligned}
$$

where $q^{2}=(u / b)^{2}+(v / c)^{2}$.

\section{Cylinder Projection Grating}

The sine and cosine cylinder projection gratings [Fig. 1(h)] are given by

$$
\begin{aligned}
& r_{1}(x, y)= \begin{cases}\sin \left(2 \pi f \sqrt{a^{2}-x^{2}}\right) & 0 \leqslant|x|<a \\
0 & a \leqslant|x|<\infty\end{cases} \\
& r_{2}(x, y)= \begin{cases}\cos \left(2 \pi f \sqrt{a^{2}-x^{2}}\right) & 0 \leqslant|x|<a \\
0 & a \leqslant|x|<\infty\end{cases}
\end{aligned}
$$

and their generating surface is the cylinder of radius $a$, $z=\sqrt{a^{2}-x^{2}}$, which is constant in the $y$ direction.

The spectrum $R_{1}(u, v)$ of $r_{1}(x, y)$ is given by the horizontal line impulse:

$$
R_{1}(u, v)=\pi f a \frac{1}{\sqrt{f^{2}+u^{2}}} J_{1}\left(2 \pi a \sqrt{f^{2}+u^{2}}\right) \delta(v) .
$$

This result can be obtained by adaptation of Eq. (37) in Ref. 19. Similarly, the spectrum of the cosinusoidal counterpart, $r_{2}(x, y)$, is given by the horizontal line impulse:

$$
R_{2}(u, v)=-\pi f a \frac{1}{\sqrt{f^{2}+u^{2}}} Y_{1}\left(2 \pi a \sqrt{f^{2}+u^{2}}\right) \delta(v)
$$

\section{GRATINGS OBTAINED FROM CONIC SURFACES, AND THEIR SPECTRA}

Owing to their straight walls, conic surfaces give gratings with constant local radial frequency in each direction.

\section{A. Circular Grating}

The cosine circular grating [Fig. 1(i)] is given by $r_{1}(x, y)$ $=\cos \left(2 \pi f \sqrt{x^{2}+y^{2}}\right)$, and its generating surface is the top-opened cone $z=\sqrt{x^{2}+y^{2}}$. In a previous paper ${ }^{1} \mathrm{I}$ showed that the spectrum $R_{1}(u, v)$ of this function is given by

$$
R_{1}(u, v)=\left\{\begin{array}{ll}
-\frac{f}{2 \pi} \frac{1}{\left(f^{2}-q^{2}\right)^{3 / 2}} & 0 \leqslant q<f \\
0 & f<q<\infty
\end{array},\right.
$$

where $q^{2}=u^{2}+v^{2}$; or, using a notation based on the half-order derivative of the Dirac impulse $\delta($ ),

$$
R_{1}(u, v)=\frac{f}{\sqrt{\pi}} \frac{1}{(f+q)^{3 / 2}} \delta^{(1 / 2)}(f-q) .
$$

This is a circular impulse ring with a particular dipolelike impulse behavior on the perimeter of a circle of ra$\operatorname{dius} f$, with a negative continuous wake, which gradually trails off towards the center. ${ }^{1}$

Similarly, the spectrum of the sine circular grating $r_{2}(x, y)=\sin \left(2 \pi f \sqrt{x^{2}+y^{2}}\right)$ is given by

$$
R_{2}(u, v)=\left\{\begin{array}{ll}
0 & 0 \leqslant q<f \\
-\frac{f}{2 \pi} \frac{1}{\left(q^{2}-f^{2}\right)^{3 / 2}} & f<q<\infty
\end{array},\right.
$$

where $q^{2}=u^{2}+v^{2}$; or, in terms of the half-order derivative of the Dirac impulse $\delta($ ),

$$
R_{2}(u, v)=\frac{f}{\sqrt{\pi}} \frac{1}{(f+q)^{3 / 2}} \delta^{(1 / 2)}(q-f) .
$$

This is a circular impulse ring with a particular dipolelike impulse behavior on the perimeter of a circle of ra$\operatorname{dius} f$, with a negative continuous wake, which surrounds it and gradually trails off outward, asymptotically to the $x, y$ plane.

\section{B. Elliptic Grating}

The cosine and sine elliptic gratings [Fig. 1(j)] are generalizations of the circular gratings that are obtained by scaling in $x$ and in $y$ :

$$
\begin{aligned}
& r_{3}(x, y)=r_{1}(b x, c y)=\cos \left[2 \pi f \sqrt{(b x)^{2}+(c y)^{2}}\right], \\
& r_{4}(x, y)=r_{2}(b x, c y)=\sin \left[2 \pi f \sqrt{(b x)^{2}+(c y)^{2}}\right] .
\end{aligned}
$$

Their generating surface is the top-opened elliptic cone $z=\sqrt{(b x)^{2}+(c y)^{2}}$.

Therefore by the $2 \mathrm{D}$ similarity theorem ${ }^{15}$ we obtain

$$
\begin{aligned}
R_{3}(u, v) & =\frac{1}{|b c|} R_{1}\left(\frac{u}{b}, \frac{v}{c}\right) \\
& = \begin{cases}-\frac{1}{|b c|} \frac{f}{2 \pi} \frac{1}{\left(f^{2}-q^{2}\right)^{3 / 2}} & 0 \leqslant q<f \\
0 & f<q<\infty\end{cases} \\
R_{4}(u, v) & =\frac{1}{|b c|} R_{2}\left(\frac{u}{b}, \frac{v}{c}\right) \\
& = \begin{cases}0 & 0 \leqslant q<f \\
-\frac{1}{|b c|} \frac{f}{2 \pi} \frac{1}{\left(q^{2}-f^{2}\right)^{3 / 2}} & f<q<\infty\end{cases}
\end{aligned}
$$

where $q^{2}=(u / b)^{2}+(v / c)^{2}$; or, in terms of the half-order derivative of the Dirac impulse $\delta()$, 


$$
\begin{aligned}
& R_{3}(u, v)=\frac{f}{\sqrt{\pi}} \frac{1}{(f+q)^{3 / 2}} \delta^{(1 / 2)}(f-q), \\
& R_{4}(u, v)=\frac{f}{\sqrt{\pi}} \frac{1}{(f+q)^{3 / 2}} \delta^{(1 / 2)}(q-f) .
\end{aligned}
$$

It is interesting to note that the intensity of the elliptic impulse ring in these spectra is higher toward the ends of the major axis of the ellipse; this phenomenon is explained for a similar case in Ref. 20 .

\section{Hyperbolic Grating}

The cosine and sine hyperbolic gratings [Fig. 1(k)], another generalization of the circular gratings, are given by

$$
\begin{aligned}
& r_{3}(x, y) \\
& \quad= \begin{cases}\cos \left[2 \pi f \sqrt{(b x)^{2}-(c y)^{2}}\right] & (b x)^{2}-(c y)^{2}>0 \\
0 & (b x)^{2}-(c y)^{2} \leqslant 0\end{cases} \\
& r_{4}(x, y) \\
& = \begin{cases}\sin \left[2 \pi f \sqrt{(b x)^{2}-(c y)^{2}}\right] & (b x)^{2}-(c y)^{2}>0 \\
0 & (b x)^{2}-(c y)^{2} \leqslant 0\end{cases}
\end{aligned}
$$

Their generating surface is the laterally oriented cone $z=\sqrt{(b x)^{2}-(c y)^{2}}$, which is opened in the $\pm x$ directions. The spectra of these functions are

$$
\begin{aligned}
R_{3}(u, v) & =\frac{1}{|b c|} R_{1}\left(\frac{u}{b}, \frac{v}{c}\right) \\
& =\left\{\begin{array}{ll}
-\frac{1}{|b c|} \frac{f}{2 \pi} \frac{1}{\left(f^{2}-q^{2}\right)^{3 / 2}} & 0 \leqslant q<f \\
0 & f<q<\infty
\end{array},\right. \\
R_{4}(u, v) & =\frac{1}{|b c|} R_{2}\left(\frac{u}{b}, \frac{v}{c}\right) \\
& =\left\{\begin{array}{ll}
0 & 0 \leqslant q<f \\
-\frac{1}{|b c|} \frac{f}{2 \pi} \frac{1}{\left(q^{2}-f^{2}\right)^{3 / 2}} & f<q<\infty
\end{array},\right.
\end{aligned}
$$

where $q^{2}=(u / b)^{2}-(v / c)^{2}$; or, in terms of the half-order derivative of the Dirac impulse $\delta($ ),

$$
\begin{aligned}
& R_{3}(u, v)=\frac{f}{\sqrt{\pi}} \frac{1}{(f+q)^{3 / 2}} \delta^{(1 / 2)}(f-q), \\
& R_{4}(u, v)=\frac{f}{\sqrt{\pi}} \frac{1}{(f+q)^{3 / 2}} \delta^{(1 / 2)}(q-f) .
\end{aligned}
$$

These spectra consist of hyperbolic curvilinear impulses that have a profile shape similar to that of their circular or elliptic counterparts (Subsections 6.A and 6.B): they have a particular dipole-like impulse behavior on their hyperbolic perimeter and a continuous wake that gradually trails off from their concave side (in the cosinusoidal grating) or from their convex side (in the sinusoidal grating).

\section{GRATINGS OBTAINED FROM HYPERBOLIC SURFACES, AND THEIR SPECTRA}

In this section we discuss gratings that are obtained from the one-sheet hyperboloid, the two-sheet hyperboloid, and the hyperbolic cylinder. Note that any hyperboloid is asymptotic to a conic surface and that any hyperbolic cylinder is asymptotic to a pair of intersecting planes. We will see below the consequences of this fact on the gratings generated by these surfaces and on their spectra.

\section{A. One-Sheet Hyperboloid Projection Grating}

The sine and cosine one-sheet hyperboloid projection gratings [Fig. 1(1)] are given by

$$
\begin{aligned}
& r_{1}(x, y)= \begin{cases}0 & 0 \leqslant r<a \\
\sin \left(2 \pi f \sqrt{r^{2}-a^{2}}\right) & a \leqslant r<\infty\end{cases} \\
& r_{2}(x, y)= \begin{cases}0 & 0 \leqslant r<a \\
\cos \left(2 \pi f \sqrt{r^{2}-a^{2}}\right) & a \leqslant r<\infty\end{cases}
\end{aligned}
$$

where $r^{2}=x^{2}+y^{2}$; their generating surface is the onesheet hyperboloid $z=\sqrt{r^{2}-a^{2}}$.

The spectrum $R_{1}(u, v)$ of $r_{1}(x, y)$ is given by

$$
\begin{array}{r}
R_{1}(u, v)=\left\{\begin{array}{l}
0 \\
\pi f a^{3 / 2} \frac{1}{\left(q^{2}-f^{2}\right)^{3 / 4}} J_{-3 / 2}\left(2 \pi a \sqrt{q^{2}-f^{2}}\right)
\end{array}\right. \\
\quad 0 \leqslant q<f, \quad f<q<\infty,
\end{array}
$$

where $q^{2}=u^{2}+v^{2}$. This result is obtained by adaptation of Eq. (24) in Ref. 21, assuming there $\nu=0$ [note that Eq. (24) is originally given there for $\nu>1 / 2$, to avoid cases with impulsive spectra]. Similarly, the spectrum of the cosinusoidal counterpart $r_{2}(x, y)$ is

$$
R_{2}(u, v)=\left\{\begin{array}{c}
-2 f a^{3 / 2} \frac{1}{\left(f^{2}-q^{2}\right)^{3 / 4}} K_{-3 / 2}\left(2 \pi a \sqrt{f^{2}-q^{2}}\right) \\
\pi f a^{3 / 2} \frac{1}{\left(q^{2}-f^{2}\right)^{3 / 4}} Y_{-3 / 2}\left(2 \pi a \sqrt{q^{2}-f^{2}}\right) \\
0 \leqslant q<f, \quad f<q<\infty,
\end{array},\right.
$$

where $K_{p}(x)$ is the modified Bessel function of the second kind of order $p$.

Note that when $a \rightarrow 0$ the gratings $r_{1}(x, y)$ and $r_{2}(x, y)$ tend to the circular sine and cosine gratings of Subsection 6.A. And indeed, it is interesting to note that when $a \rightarrow 0$ the spectra $R_{1}(u, v)$ and $R_{2}(u, v)$ tend to the spectra of the circular sine and cosine gratings [Eqs. (4) and (3) in Subsection 6.A]. This can be verified by replacing the Bessel functions inside $R_{1}(u, v)$ and $R_{2}(u, v)$ with their series developments ${ }^{18}$ and proceeding to the limit.

B. Two-Sheet Hyperboloid Projection Grating

The sine and cosine two-sheet hyperboloid projection gratings [Fig. 1(m)] are given by $r_{1}(x, y)$ $=\sin \left(2 \pi f \sqrt{r^{2}+a^{2}}\right)$ and $r_{2}(x, y)=\cos \left(2 \pi f \sqrt{r^{2}+a^{2}}\right)$, where $r^{2}=x^{2}+y^{2}$, and their generating surface is the one-sheet hyperboloid $z=\sqrt{r^{2}+a^{2}}$. 
The spectrum $R_{1}(u, v)$ of $r_{1}(x, y)$ is given by

$$
R_{1}(u, v)=\left\{\begin{array}{c}
-\pi f a^{3 / 2} \frac{1}{\left(f^{2}-q^{2}\right)^{3 / 4}} J_{3 / 2}\left(2 \pi a \sqrt{f^{2}-q^{2}}\right) \\
-2 f a^{3 / 2} \frac{1}{\left(q^{2}-f^{2}\right)^{3 / 4}} K_{3 / 2}\left(2 \pi a \sqrt{q^{2}-f^{2}}\right) \\
0 \leqslant f, \quad f<q<\infty,
\end{array}\right.
$$

where $q^{2}=u^{2}+v^{2}$. Similarly, the spectrum of the cosinusoidal counterpart $r_{2}(x, y)$ is

$$
\begin{aligned}
& R_{2}(u, v)=\left\{\begin{array}{l}
\pi f a^{3 / 2} \frac{1}{\left(f^{2}-q^{2}\right)^{3 / 4}} Y_{3 / 2}\left(2 \pi a \sqrt{f^{2}-q^{2}}\right) \\
0
\end{array},\right. \\
& 0 \leqslant q<f, \quad f<q<\infty .
\end{aligned}
$$

This result is obtained by adaptation of Eq. (43) in Ref. 22, assuming there $\nu=0$ [note that Eq. (43) is originally given there for $-1<\nu<-1 / 2$ to avoid cases with impulsive spectra]. Note that the remark at the end of Subsection 7.A applies here, too.

\section{Laterally Opened Hyperbolic-Cylinder Projection Grating}

The sinusoidal and cosinusoidal versions of this grating [Fig. 1(n)] are given by

$$
\begin{aligned}
& r_{1}(x, y)= \begin{cases}0 & 0 \leqslant|x|<a \\
\sin \left(2 \pi f \sqrt{x^{2}-a^{2}}\right) & a \leqslant|x|<\infty\end{cases} \\
& r_{2}(x, y)= \begin{cases}0 & 0 \leqslant|x|<a \\
\cos \left(2 \pi f \sqrt{x^{2}-a^{2}}\right) & a \leqslant|x|<\infty\end{cases}
\end{aligned}
$$

and their generating surface is the laterally opened hyperbolic cylinder $z=\sqrt{x^{2}-a^{2}}$, which is constant in the $y$ direction.

The spectrum $R_{1}(u, v)$ of $r_{1}(x, y)$ is given by the horizontal line impulse

$$
R_{1}(u, v)=\left\{\begin{array}{c}
2 f a \frac{1}{\sqrt{f^{2}-u^{2}}} K_{-1}\left(2 \pi a \sqrt{f^{2}-u^{2}}\right) \delta(v) \\
-\pi f a \frac{1}{\sqrt{u^{2}-f^{2}}} Y_{-1}\left(2 \pi a \sqrt{u^{2}-f^{2}}\right) \delta(v) \\
0 \leqslant u<f, \quad f<u<\infty .
\end{array}\right.
$$

Similarly, the spectrum of the cosinusoidal counterpart $r_{2}(x, y)$ is given by the horizontal line impulse:

$$
\begin{array}{r}
R_{2}(u, v)=\left\{\begin{array}{l}
0 \\
\pi f a \frac{1}{\sqrt{u^{2}-f^{2}}} J_{-1}\left(2 \pi a \sqrt{u^{2}-f^{2}}\right) \delta(v)
\end{array}\right. \\
\quad 0 \leqslant u<f, \quad f<u<\infty .
\end{array}
$$

Note that when $a \rightarrow 0$ the gratings $r_{1}(x, y)$ and $r_{2}(x, y)$ tend to $\sin (2 \pi f|x|)$ and $\cos (2 \pi f x)$, respectively. And indeed, when $a \rightarrow 0$ the spectra $R_{1}(u, v)$ and $R_{2}(u, v)$ tend to the spectra of these functions, which are, respectively, $\quad(f / \pi)\left[1 /\left(f^{2}-u^{2}\right)\right]$ and $(1 / 2) \delta(x-f)$ $+(1 / 2) \delta(x+f)$. This can be verified, again, by replacing the Bessel functions inside $R_{1}(u, v)$ and $R_{2}(u, v)$ with their series developments ${ }^{18}$ and proceeding to the limit.

\section{Top-Opened Hyperbolic-Cylinder Projection Grating}

The sinusoidal and cosinusoidal versions of this grating [Fig. 1(o)] are given by $r_{1}(x, y)=\sin \left(2 \pi f \sqrt{x^{2}+a^{2}}\right)$ and $r_{2}(x, y)=\cos \left(2 \pi f \sqrt{x^{2}+a^{2}}\right)$, and their generating surface is the top-opened hyperbolic cylinder $z=\sqrt{x^{2}+a^{2}}$, which is constant in the $y$ direction.

The spectrum $R_{1}(u, v)$ of $r_{1}(x, y)$ is given by the horizontal line impulse:

$$
R_{1}(u, v)=\left\{\begin{array}{c}
-\pi f a \frac{1}{\sqrt{f^{2}-u^{2}}} Y_{1}\left(2 \pi a \sqrt{f^{2}-u^{2}}\right) \delta(v) \\
-2 f a \frac{1}{\sqrt{u^{2}-f^{2}}} K_{1}\left(2 \pi a \sqrt{u^{2}-f^{2}}\right) \delta(v) \\
0 \leqslant u<f, \quad f<u<\infty .
\end{array}\right.
$$

This result is obtained from formula 5.93 in Ref. 23, as suming there $v=1$ (note that formula 5.93 is originally given there for $-1<v<1$ to avoid cases with impulsive spectra). Similarly, the spectrum of the cosinusoidal counterpart $r_{2}(x, y)$ is given by the horizontal line impulse:

$$
\begin{aligned}
& R_{2}(u, v)=\left\{\begin{array}{l}
-\pi f a \frac{1}{\sqrt{f^{2}-u^{2}}} J_{1}\left(2 \pi a \sqrt{f^{2}-u^{2}}\right) \delta(v) \\
0
\end{array},\right. \\
& 0 \leqslant u<f, \quad f<u<\infty .
\end{aligned}
$$

This result is obtained from formula 5.94 in Ref. 23, assuming there $v=1$. Note that the remark at the end of Subsection 7.C applies here, too.

\section{E. Other Cases}

Finally, it should be noted that more general second-order curvilinear gratings can be obtained from each of the above cases by translations, rotations, shears, or any other first-order coordinate transformations of the $x, y$ plane. The influence of a first-order transformation on the Fourier domain is well known and rather straightforward. ${ }^{24}$ Some additional interesting cases that are obtained by other transformations are given in the appendices.

\section{SPECTRUM OF CURVILINEAR GRATINGS WITH ANY GIVEN PERIODIC PROFILE}

\section{A. Fourier Decomposition of Curvilinear Gratings}

The analysis of curvilinear gratings with any arbitrary periodic profile is based on the Fourier series decomposition of the gratings' periodic profile. Assume that the curvilinear grating $r(x, y)$ is obtained by bending a periodic grating $p\left(x^{\prime}\right)$, i.e., by replacing $x^{\prime}$ by a function $x^{\prime}=g_{1}(x, y): \quad r(x, y)=p\left[g_{1}(x, y)\right]$. A few examples 
$r(x, y)$
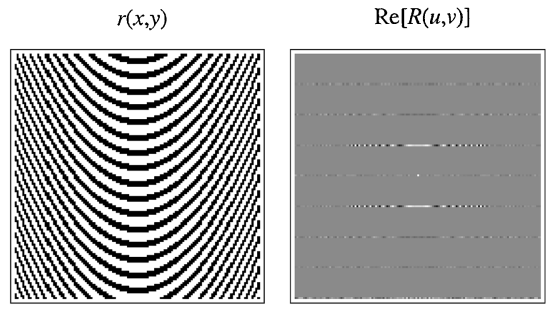

(a)
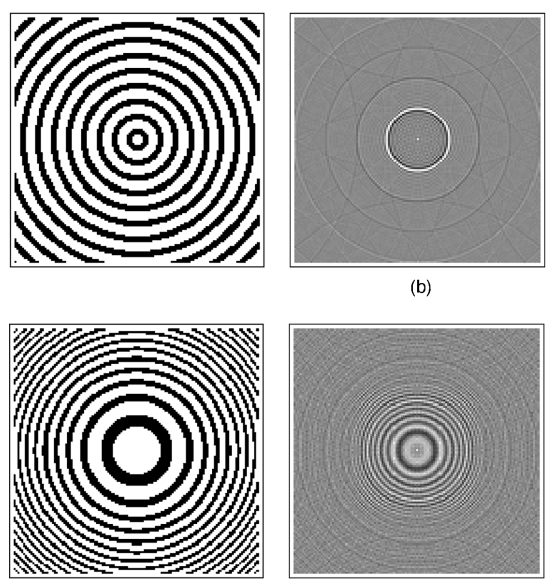

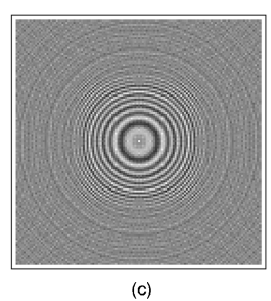

(c)
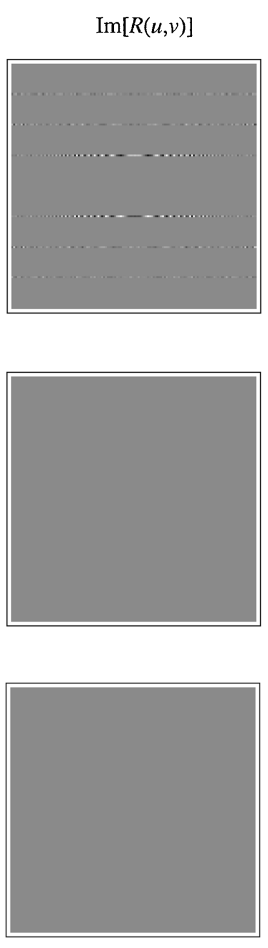

Fig. 3. Some examples of curvilinear gratings $r(x, y)$ with a square-wave periodic profile (with opening ratio $\tau / T=0.6$ ), and their respective spectra $R(u, v)$ : (a) parabolic grating, $g_{1}(x, y)=y-0.15 x^{2} ; \quad$ (b) circular grating, $g_{1}(x, y)$ $=\sqrt{x^{2}+y^{2}}$; (c) circular zone grating, $g_{1}(x, y)=\left(x^{2}+y^{2}\right) / 8$. The amplitudes of the different harmonics in the spectra are weighted by the Fourier series coefficients $a_{n}$ of the square wave [see Eq. (12)]: $a_{1}=0.303, a_{2}=-0.094, a_{3}=-0.062$, etc; the sign inversions in the second and third harmonics are clearly visible in the spectra of cases (a) and (b). Notice the various DFT artifacts in the spectra (folding over due to aliasing; rippling).

of curvilinear gratings $r(x, y)$ with a square-wave periodic-profile $p\left(x^{\prime}\right)$ are shown in Fig. 3. If the Fourier development of the original grating $p\left(x^{\prime}\right)$ is

$$
p\left(x^{\prime}\right)=\sum_{n=-\infty}^{\infty} c_{n} \exp \left(i 2 \pi n f x^{\prime}\right)
$$

then the Fourier decomposition of our curvilinear grating $r(x, y)$ is ${ }^{25}$

$$
r(x, y)=p\left[g_{1}(x, y)\right]=\sum_{n=-\infty}^{\infty} c_{n} \exp \left[i 2 \pi n f g_{1}(x, y)\right] .
$$

Note that the exponential form of the Fourier series [Eq. (5)] can be equivalently expressed in terms of cosine and sine series as follows:

$$
\begin{aligned}
p\left(x^{\prime}\right)= & a_{0}+2 \sum_{n=1}^{\infty} a_{n} \cos \left(2 \pi n x^{\prime} / T\right) \\
& +2 \sum_{n=1}^{\infty} b_{n} \sin \left(2 \pi n x^{\prime} / T\right)
\end{aligned}
$$

where $a_{0}=c_{0}, \quad a_{n}=\left(c_{n}+c_{-n}\right) / 2, \quad b_{n}=i\left(c_{n}-c_{-n}\right) / 2$ $(n \geqslant 1)$, and $T=1 / f$; and vice versa, knowing the cosine and sine Fourier decomposition (7), one obtains the exponential form (5) by taking $c_{0}=a_{0}, c_{n}=a_{n}-i b_{n}, c_{-n}$ $=a_{n}+i b_{n}(n \geqslant 1)$.

In the special case where $p\left(x^{\prime}\right)$ is symmetric, the series developments (5) and (7) reduce into a pure cosine development:

$$
p\left(x^{\prime}\right)=a_{0}+2 \sum_{n=1}^{\infty} a_{n} \cos \left(2 \pi n x^{\prime} / T\right),
$$

and Eq. (6) becomes

$$
\begin{aligned}
r(x, y) & =p\left[g_{1}(x, y)\right] \\
& =a_{0}+2 \sum_{n=1}^{\infty} a_{n} \cos \left(2 \pi n g_{1}(x, y) / T\right)
\end{aligned}
$$

with the same coefficients $a_{n}$ as in Eq. (8). This cosine series development lends itself more easily to graphic interpretation. However, it cannot be used for gratings with nonsymmetric periodic-profile forms such as sawtooth waves, and in such cases the general exponential development (5) (or equivalently, the development (7) into cosine and sine series) must be used.

\section{B. Spectrum of Curvilinear Gratings}

We have seen in Subsection 8.A that a curvilinear grating $r(x, y)$ can be represented in the image domain as a curvilinear Fourier series, i.e., as a sum of curvilinear cosines and sines (or exponentials) that were all subjected to the same transformation $g_{1}(x, y)$ as the curvilinear grating $r(x, y)$ itself. This fact reduces the problem of finding $R(u, v)$, the spectrum of the curvilinear grating $r(x, y)$, into the question of finding the Fourier transform of the curvilinear cosine and sine (or exponential). In the classical case of periodic functions, the Fourier transform pair

$$
\cos (2 \pi f x) \leftrightarrow \frac{1}{2} \delta(u-f)+\frac{1}{2} \delta(u+f)
$$

gives us

$$
\begin{aligned}
p(x) & =\sum_{n=-\infty}^{\infty} a_{n} \cos (2 \pi n x / T) \\
& \leftrightarrow P(u)=\sum_{n=-\infty}^{\infty} a_{n} \delta(u-n / T) .
\end{aligned}
$$

In a similar way, if we know here the spectrum $R_{n}(u, v)$ of the curvilinear cosine $r_{n}(x, y)=\cos \left(2 \pi n g_{1}(x, y) / T\right)$, we obtain from Eq. (9)

$$
\begin{aligned}
r(x, y) & =\sum_{n=-\infty}^{\infty} a_{n} \cos \left(2 \pi n g_{1}(x, y) / T\right) \\
& \leftrightarrow R(u, v)=\sum_{n=-\infty}^{\infty} a_{n} R_{n}(u, v) .
\end{aligned}
$$

Similarly, using the more general exponential notation, we obtain from Eq. (6) 


$$
\begin{aligned}
r(x, y) & =\sum_{n=-\infty}^{\infty} c_{n} \exp \left[i 2 \pi n f g_{1}(x, y)\right] \\
& \leftrightarrow R(u, v)=\sum_{n=-\infty}^{\infty} c_{n} R_{n}(u, v),
\end{aligned}
$$

where $R_{n}(u, v)$ is the spectrum of the curvilinear exponential function $r_{n}(x, y)=\exp \left[i 2 \pi n f g_{1}(x, y)\right]$.

In other words, the spectrum of the curvilinear grating $r(x, y)$ is the sum of the spectra of the individual curvilinear cosines and sines (or exponentials), where $a_{n}, b_{n}$ (or $c_{n}$ ) are the same coefficients as in the Fourier series decomposition of $r(x, y)$ and hence, according to Subsection 8.A, the same coefficients as in the decomposition of its periodic profile $p\left(x^{\prime}\right)$.

Therefore, to investigate the spectrum of the curvilinear grating $r(x, y)$ we first have to understand what happens to the spectra of the $2 \mathrm{D}$ cosine and sine functions $\cos (2 \pi f x)$ and $\sin (2 \pi f x)$ [or, equivalently, to the spectrum of an exponential function $\exp (i 2 \pi f x)]$ when the image domain undergoes a transformation (or coordinate change) $g_{1}(x, y)$. Unfortunately, there exists no such general rule when the transformation $g_{1}(x, y)$ is nonlinear. However, for many cases of interest the Fourier transform of $\cos \left[2 \pi f g_{1}(x, y)\right]$ and $\sin \left[2 \pi f g_{1}(x, y)\right]$ (or of $\left.\exp \left[i 2 \pi f g_{1}(x, y)\right]\right)$ can be found on the basis of Fourier transform tables in the literature (such as in Refs. 17, 19, 21 , and 23) that include Fourier transforms of functions of the forms $\cos [g(x)], \sin [g(x)]$, or $\exp [g(x)]$. Some particular cases of interest have been discussed above in Sections 4-7 and illustrated in Fig. 1. As we have seen, in some cases (cylinder projection gratings: Subsections 4.D, 5.C, etc.) the spectra of the curvilinear cosine and sine functions are impulsive, whereas in other situations their spectra may become semi-impulsive (Section 6) or even completely nonimpulsive (e.g., Subsections 4.A-4.C).

Equations (10) and (11) are particularly useful when the individual spectra $R_{n}(u, v)$ of the curvilinear cosines (or exponentials) are impulsive. In this case the spectrum $R(u, v)$ is composed of isolated, separately localized entities (impulses), and each of the terms $R_{n}(u, v)$ in the series represents indeed one of these isolated entities in the spectrum. If the individual spectra $R_{n}(u, v)$ are continuous [as in the case of Figs. 1(b)-(d)], Eqs. (10) and (11) are still valid-but they lose much of their practical usefulness: In this case the spectrum $R(u, v)$ $=\sum_{n=-\infty}^{\infty} a_{n} R_{n}(u, v)$ is a sum of continuous functions with overlapping supports, and its series representation no longer reflects a partition of the spectrum into spatially separated entities $R_{n}(u, v)$ with mutually exclusive supports on the $u, v$ plane that can be individually localized, isolated, and manipulated. Nevertheless, in many cases one can still make use of such continuous spectra, for example in questions involving the synthesis or reconstruction of the functions $R(u, v)$ and $r(x, y)$.

Example 1. The spectrum of a parabolic grating with a square-wave periodic profile:

We recall that the Fourier series decomposition of the square wave $p\left(x^{\prime}\right)$ with period $T$ and opening $\tau$ is

$$
p\left(x^{\prime}\right)=\sum_{n=-\infty}^{\infty} a_{n} \cos \left(2 \pi n x^{\prime} / T\right)
$$

with

$$
a_{n}=(\tau / T) \operatorname{sinc}(n \tau / T) .
$$

Now let $r(x, y)$ be a parabolic grating that has the square-wave periodic profile $p\left(x^{\prime}\right)$ with period $T$ and opening $\tau$ [see Fig. 3(a)]. This curvilinear grating is obtained by bending $p\left(x^{\prime}\right)$, i.e., by applying on $p\left(x^{\prime}\right)$ the nonlinear transformation (or coordinate change) $x^{\prime}$ $=y-a x^{2}$. The Fourier decomposition of $r(x, y)$ is obtained by replacing $x^{\prime}$ with $y-a x^{2}$ in Eq. (12):

$$
r(x, y)=p\left(y-a x^{2}\right)=\sum_{n=-\infty}^{\infty} a_{n} \cos \left(2 \pi n\left(y-a x^{2}\right) / T\right) .
$$

Therefore the spectrum of this grating is

$$
R(u, v)=\sum_{n=-\infty}^{\infty} a_{n} R_{n}(u, v)
$$

where $R_{0}(u, v)=\delta(u, v)$ is the dc impulse and $R_{n}(u, v)$, the spectrum of $\cos \left[2 \pi n\left(y-a x^{2}\right) / T\right]$, is a pair of continuous horizontal line impulses that are vertically located at $v= \pm n / T$ (see Subsection A.1 in Appendix A):

$$
\begin{aligned}
R_{n}(u, v)= & \frac{1}{2}\left[R_{c}(u)+i R_{s}(u)\right] \delta(v-n f) \\
& +\frac{1}{2}\left[R_{c}(u)-i R_{s}(u)\right] \delta(v+n f),
\end{aligned}
$$

with

$$
\begin{aligned}
& R_{c}(u)=\frac{1}{2 \sqrt{n f a}}\left[\cos \left(\frac{\pi}{2 n f a} u^{2}\right)+\sin \left(\frac{\pi}{2 n f a} u^{2}\right)\right], \\
& R_{s}(u)=\frac{1}{2 \sqrt{n f a}}\left[\cos \left(\frac{\pi}{2 n f a} u^{2}\right)-\sin \left(\frac{\pi}{2 n f a} u^{2}\right)\right],
\end{aligned}
$$

where $a$ is the bending rate of the parabolic grating $r(x, y)$ and $f$ is its fundamental frequency $1 / T$.

The spectrum of the parabolic grating with a squarewave periodic profile consists, therefore, of a dc impulse plus a series of such horizontal line impulses that are vertically situated at $v=n / T, n= \pm 1, \pm 2, \ldots$, and whose amplitudes are weighted by the coefficients $a_{n}$ [see Fig. $3(\mathrm{a})$.

Example 2. The spectrum of a circular grating with a square-wave periodic profile:

The Fourier decomposition of the circular grating $r(x, y)$ into a series of circular cosines with radial frequencies of $f_{n}=n / T(=$ radial periods of $T / n)$ is

$$
\begin{aligned}
r(x, y) & =p\left(\sqrt{x^{2}+y^{2}}\right) \\
& =a_{0}+2 \sum_{n=1}^{\infty} a_{n} \cos \left(2 \pi n \sqrt{x^{2}+y^{2}} / T\right)
\end{aligned}
$$

with the same coefficients $a_{n}$ as in the square-wave profile: $a_{n}=(\tau / T) \operatorname{sinc}(n \tau / T)$. Therefore the spectrum of this circular grating is

$$
R(u, v)=a_{0} R_{0}(u, v)+2 \sum_{n=1}^{\infty} a_{n} R_{n}(u, v),
$$


where $R_{0}(u, v)$ is the dc impulse and $R_{n}(u, v)$, the spectrum of $\cos \left(2 \pi n \sqrt{x^{2}+y^{2}} / T\right)$, is a peculiar dipole-like impulse ring with a weak continuous wake trailing off toward the center, as we have seen in Subsection 6.A (see Ref. 1 for more details). The spectrum of a circular grating with a square-wave periodic profile is, therefore, a concentric series of such circular dipole-like rings with radii of $n / T$, whose amplitudes are weighted by the coefficients $a_{n}$ of the square-wave profile [see Fig. 3(b)]. Note that in this case the rings $R_{n}(u, v)$ are not completely spatially separable, since their weak, continuous wakes, which trail off toward the spectrum center, are overlapping. However, for many practical needs these continuous wakes can be considered negligible, and we can say that the main frequency contribution of each of the rings $R_{n}(u, v)$ is concentrated on its impulsive ( = singular) support, namely, on the perimeter of a circle with radius $n / T$ around the spectrum origin. Note, however, that even on this singular support the impulsive behavior of $R_{n}(u, v)$ is dipole-like and hence more complex than that of a simple impulse ring $\delta\left(\sqrt{u^{2}+v^{2}}-f\right)$.

Example 3. The spectrum of a zone grating with square-wave periodic profile:

As we have seen in Section 4, a zone grating (zone plate) is a concentric circular grating where the radius of the $n$th circle is proportional to $\sqrt{n}$. In most optical applications the periodic profile of the zone grating has a binary (black/white) square-wave form. The Fourier development of this function is therefore [see Fig. 3(c)]

$$
r(x, y)=a_{0}+2 \sum_{n=1}^{\infty} a_{n} \cos \left[2 \pi n\left(x^{2}+y^{2}\right) / T\right]
$$

with the same coefficients $a_{n}$ as in the square-wave profile: $a_{n}=(\tau / T) \operatorname{sinc}(n \tau / T)$. Therefore the spectrum of this circular grating is

$$
R(u, v)=a_{0} R_{0}(u, v)+2 \sum_{n=1}^{\infty} a_{n} R_{n}(u, v),
$$

where $R_{0}(u, v)$ is the dc impulse $\delta(u, v)$ and $R_{n}(u, v)$, the spectrum of $\cos \left(2 \pi n\left(x^{2}+y^{2}\right) / T\right)$, is, according to Subsection 4.A, $R_{n}(u, v)=\frac{1}{2 n f} \sin \left[\frac{\pi}{2 n f}\left(u^{2}+v^{2}\right)\right]$, where $f$ is the fundamental frequency $1 / T$. Note that the terms $R_{n}(u, v)$ of the spectrum $R(u, v)$ are not spatially separable, since each of them is a continuous sinusoidal zone grating that is centered on the origin and extends throughout the whole spectrum, and hence all of them are mutually overlapping at every point of the $u, v$ plane.

\section{SUMMARY}

Second-order curvilinear gratings are mathematically obtained by the application of a second-order spatial transformation on a straight, periodic grating. Such curvilinear structures occur quite frequently in optics (circular gratings, various zone plates, etc.), and their Fourier transform may arise, for example, in connection with the Fraunhofer diffraction patterns generated by these structures. However, owing to the nonlinearity of the spatial transformations involved, the analytic expressions of the Fourier transforms of most second-order curvilinear gratings are not easy to find.

In this paper are provided the analytic spectra of the most important families of second-order curvilinear gratings, and their main properties are shown. We started with second-order curvilinear gratings with a cosinusoidal or a sinusoidal intensity profile. Then we generalized these results for curvilinear gratings with any desired intensity profiles, using the Fourier decomposition of their intensity profile. These results open the way to a better understanding of the properties of many important curvilinear gratings and their Fourier spectra.

\section{APPENDIX A: EFFECTS OF THE TRANSFORMATION $g(x, y)=g_{1}(x, y)$ $+b x+c y$}

Let $g_{1}(x, y)$ be the bending transformation of the curvilinear gratings $r_{1}(x, y)=\cos \left[2 \pi f g_{1}(x, y)\right]$ and $r_{2}(x, y)$ $=\sin \left[2 \pi f g_{1}(x, y)\right]$; the generating surface associated with this bending transformation is $z=g_{1}(x, y)$. Assume that we add to $g_{1}(x, y)$ a term of the form $b x, c y$ or $b x+c y$; this is equivalent to an incrementation of the generating surface $z=g_{1}(x, y)$ by a plane $z=b x$, $z=c y$ or $z=b x+c y$, namely, a linear inclination of the generating surface in the $x$ direction, in the $y$ direction, or in both. How does this affect the spectrum of the gratings $r_{1}(x, y)$ and $r_{2}(x, y)$ ?

Using the trigonometric identity $\cos (\alpha+\beta)$

$=\cos \alpha \cos \beta-\sin \alpha \sin \beta$, we obtain

$$
\begin{aligned}
r_{3}(x, y)= & \cos \left\{2 \pi f\left[g_{1}(x, y)+(b x+c y)\right]\right\} \\
= & \cos \left[2 \pi f g_{1}(x, y)\right] \cos [2 \pi f(b x+c y)] \\
& -\sin \left[2 \pi f g_{1}(x, y)\right] \sin [2 \pi f(b x+c y)] .
\end{aligned}
$$

However, according to the $2 \mathrm{D}$ modulation theorem, ${ }^{15}$ we have

$$
\begin{aligned}
& r_{1}(x, y) \cos [2 \pi f(b x+c y)] \\
& \leftrightarrow \frac{1}{2} R_{1}(u+b f, v+c f)+\frac{1}{2} R_{1}(u-b f, v-c f), \\
& r_{2}(x, y) \sin [2 \pi f(b x+c y)] \\
& \leftrightarrow \frac{1}{2} i R_{2}(u+b f, v+c f)-\frac{1}{2} i R_{2}(u-b f, v-c f),
\end{aligned}
$$

where $R_{1}(u, v)$ is the spectrum of $r_{1}(x, y)$ and $R_{2}(u, v)$ is the spectrum of $r_{2}(x, y)$. Therefore the spectrum of the grating $r_{3}(x, y)$ is

$$
\begin{aligned}
& R_{3}(u, v) \\
& \quad=\frac{1}{2}\left[R_{1}(u+b f, v+c f)+R_{1}(u-b f, v-c f)\right] \\
& \quad+\frac{1}{2} i\left[R_{2}(u+b f, v+c f)-R_{2}(u-b f, v-c f)\right]
\end{aligned}
$$

Similarly, the spectrum of the grating $r_{4}(x, y)$ $=\sin \left\{2 \pi f\left[g_{1}(x, y)+(b x+c y)\right]\right\}$ is

$$
\begin{aligned}
& R_{4}(u, v) \\
& \quad=\frac{1}{2}\left[R_{2}(u+b f, v+c f)+R_{2}(u-b f, v-c f)\right] \\
& \quad+\frac{1}{2} i\left[R_{1}(u+b f, v+c f)+R_{1}(u-b f, v-c f)\right] .
\end{aligned}
$$


We obtain, therefore, the following general result:

Result 1. If the spectra of the curvilinear gratings $r_{1}(x, y)=\cos \left[2 \pi f g_{1}(x, y)\right] \quad$ and $r_{2}(x, y)$ $=\sin \left[2 \pi f g_{1}(x, y)\right]$ are, respectively, $R_{1}(u, v)$ and $R_{2}(u, v)$, then

(a) The spectrum $R_{3}(u, v)$ of the grating $r_{3}(x, y)$ $=\cos \left\{2 \pi f\left[g_{1}(x, y)+b x+c y\right]\right\}$ consists of a copy of $\frac{1}{2}\left[R_{1}(u, v)+i R_{2}(u, v)\right]$ that is shifted to the point $(u, v)=(b f, c f)$ and a copy of $\frac{1}{2}\left[R_{1}(u, v)\right.$ - $\left.i R_{2}(u, v)\right]$ that is shifted to the symmetric point $(u, v)=-(b f, c f)$.

(b) The spectrum $R_{4}(u, v)$ of the grating $r_{4}(x, y)$ $=\sin \left\{2 \pi f\left[g_{1}(x, y)+b x+c y\right]\right\}$ consists of a copy of $\frac{1}{2}\left[R_{2}(u, v)+i R_{1}(u, v)\right]$ that is shifted to the point $(u, v)=(b f, c f)$ and a copy of $\frac{1}{2}\left[R_{2}(u, v)\right.$ $\left.+i R_{1}(u, v)\right]$ that is shifted to the symmetric point $(u, v)=-(b f, c f)$

$r(x, y)$
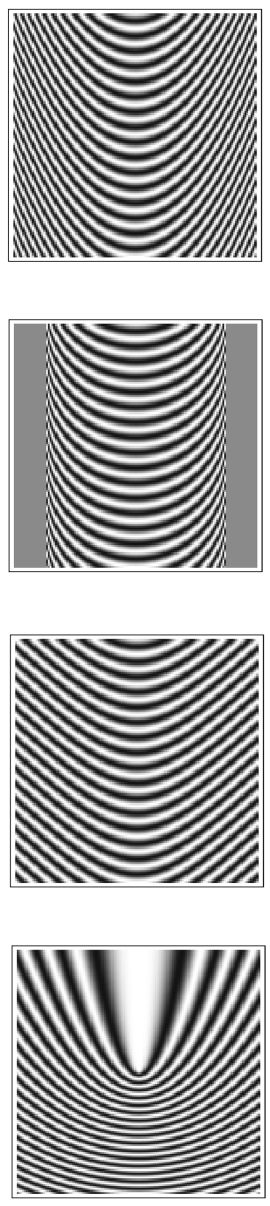

$\operatorname{Re}[R(u, v)]$

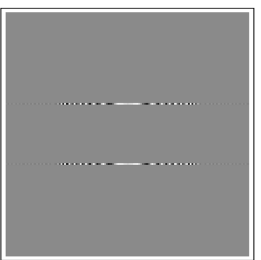

(a)

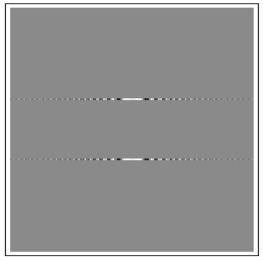

(b)

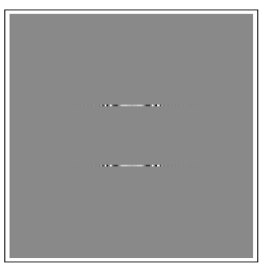

(c)

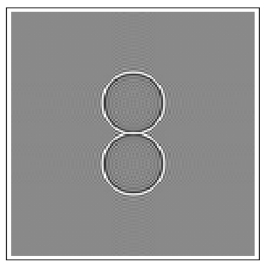

(d)
Several examples that give rise to interesting curvilinear gratings are given in the subsections that follow.

\section{Equispaced Parabolic Grating}

Assume that we add the term $-y$ to the generating surface $z=a x^{2}$ of the linear zone grating (Subsection 4.D). We obtain the generating surface $z=a x^{2}-y$, which is an inclined version of the original parabolic cylinder. The level lines $z=n(n \in \mathbb{Z})$ of this surface consist of a series of equispaced parabolas $y=a x^{2}-n$, and therefore the generated gratings $r_{5}(x, y)=\cos \left[2 \pi f\left(a x^{2}-y\right)\right]$ and $r_{6}(x, y)=\sin \left[2 \pi f\left(a x^{2}-y\right)\right]$ are equispaced parabolic gratings [Fig. 4(a)].

Now, according to result 1 , the Fourier spectrum of grating $r_{5}(x, y)$ is

$$
\begin{aligned}
R_{5}(u, v)=\frac{1}{2}\left[R_{1}(u, v-f)+R_{1}(u, v+f)\right] \\
+\frac{1}{2} i\left[R_{2}(u, v-f)-R_{2}(u, v+f)\right],
\end{aligned}
$$
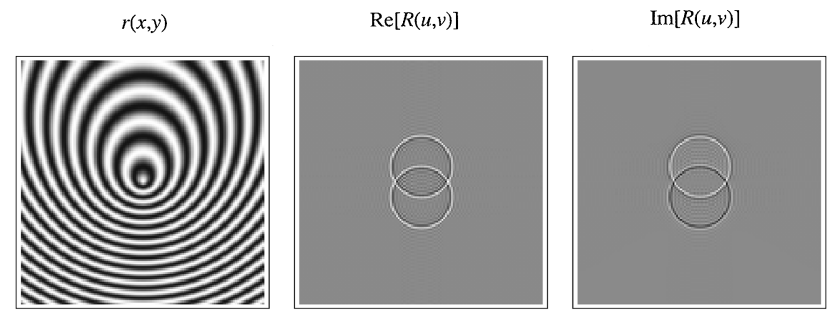

(e)
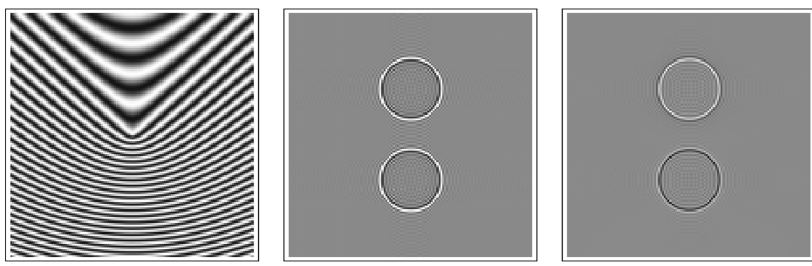

(f)
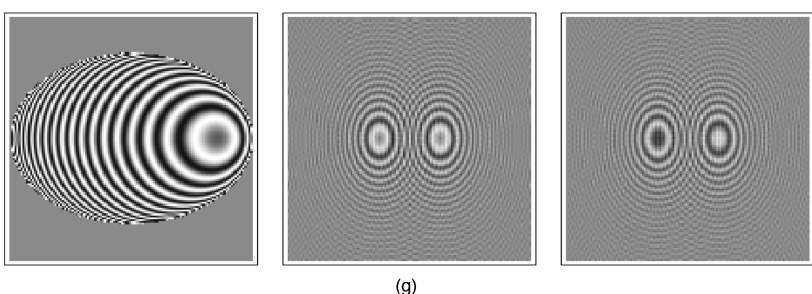

Fig. 4. Some further curvilinear gratings $r(x, y)$ with a periodic-profile waveform of $\cos (2 \pi f x)$ (with $f=1$ ), and their spectra $R(u, v$ ) as obtained on computer by 2D DFT: (a) equispaced parabolic grating, cos $\left[2 \pi f\left(0.15 x^{2}-y\right)\right]$; (b) equispaced circle-arc grating, $\cos \left[2 \pi f\left(\sqrt{36-x^{2}}+y\right)\right]$; (c) equispaced hyperbola-arc grating, $\cos \left[2 \pi f\left(\sqrt{x^{2}+16}-y\right)\right]$; (d) eccentric equispaced parabolic grating, $\cos \left[2 \pi f\left(\sqrt{x^{2}+y^{2}}-y\right)\right]$; (e) eccentric equispaced elliptic grating, $\cos \left[2 \pi f\left(\sqrt{x^{2}+y^{2}}-y / 2\right)\right]$; (f) eccentric equispaced hyperbolic grating, $\cos \left[2 \pi f\left(\sqrt{x^{2}+y^{2}}-1.5 y\right)\right]$; $(\mathrm{g})$ eccentric ellipsoid projection grating, $\cos \left[2 \pi f\left(\sqrt{64-x^{2}-2 y^{2}}+x\right)\right]$. In each case the central column shows the real part of the spectrum, and the right-hand column shows the imaginary part of the spectrum. Note the DFT rippling artifacts in some of the spectra. 
and by inserting the spectra $R_{1}(u, v)$ and $R_{2}(u, v)$ from Subsection 4.D,

$$
\begin{aligned}
=\frac{1}{2} & {\left[R_{c}(u)+i R_{s}(u)\right] \delta(v-f) } \\
& +\frac{1}{2}\left[R_{c}(u)-i R_{s}(u)\right] \delta(v+f),
\end{aligned}
$$

where $R_{c}(u)$ and $R_{s}(u)$ are given by Eqs. (1) and (2). As we can see in Fig. 4(a), this spectrum consists, indeed, of a pair of continuous horizontal line impulses ("blades"), situated at a distance of $\pm f$ from the $u$ axis. Their continuous, modulated amplitudes are given by $\frac{1}{2}\left[R_{c}(u)\right.$ $\left.+i R_{s}(u)\right]$ and $\frac{1}{2}\left[R_{c}(u)-i R_{s}(u)\right]$, respectively.

Similarly, the spectrum of the sinusoidal grating $r_{6}(x, y)$ is

$$
\begin{aligned}
R_{6}(u, v)= & \frac{1}{2}\left[R_{s}(u)+i R_{c}(u)\right] \delta(v-f) \\
& +\frac{1}{2}\left[R_{s}(u)+i R_{c}(u)\right] \delta(v+f) .
\end{aligned}
$$

2. Equispaced Circle- and Hyperbola-Arc Gratings In a similar way, if we add the term $y$ to the generating surface $z=\sqrt{a^{2}-x^{2}}$ of the cylinder projection gratings $r_{1}(x, y)$ and $r_{2}(x, y)$ of Subsection 5.C, we obtain the equispaced circle-arc gratings [Fig. 4(b)]:

$$
\begin{aligned}
& r_{5}(x, y)=\left\{\begin{array}{ll}
\sin \left[2 \pi f\left(\sqrt{a^{2}-x^{2}}+y\right)\right] & 0 \leqslant|x|<a \\
0 & a \leqslant|x|<\infty
\end{array},\right. \\
& r_{6}(x, y)=\left\{\begin{array}{ll}
\cos \left[2 \pi f\left(\sqrt{a^{2}-x^{2}}+y\right)\right] & 0 \leqslant|x|<a \\
0 & a \leqslant|x|<\infty
\end{array} .\right.
\end{aligned}
$$

Their spectra consist, again, of a pair of horizontal line impulses situated at a distance of $\pm f$ from the $u$ axis; their modulated amplitudes are determined, according to result 1 , by the spectra $R_{1}(u, v)$ and $R_{2}(u, v)$ of Subsection 5.C.

Similarly, by adding the term $y$ to the generating surface of the hyperbolic-cylinder projection grating (Subsection 7.D), one gets in the image domain an equispaced hyperbola-arc grating [Fig. 4(c)]. Its spectrum consists, again, of a pair of horizontal line impulses.

\section{Eccentric Equispaced Parabolic, Elliptic, and Hyperbolic Gratings}

Assume that we add the term $b x+c y$ to the generating surface $z=\sqrt{x^{2}+y^{2}}$ of the circular grating (Subsection 6.A). We obtain the generating surface $z=\sqrt{x^{2}+y^{2}}$ $+b x+c y$, which is an inclined version of the original top-opened cone. The level lines $z=n(n \in \mathbb{Z})$ of this surface, and hence also the resulting gratings $r_{5}(x, y)$ $=\sin \left[2 \pi f\left(\sqrt{x^{2}+y^{2}}+b x+c y\right)\right]$ and $r_{6}(x, y)$ $=\cos \left[2 \pi f\left(\sqrt{x^{2}+y^{2}}+b x+c y\right)\right]$, consist of a series of eccentric equispaced parabolas, ellipses, or hyperbolas, depending on the parameters $b$ and $c$ [see Figs. 4(d), 4(e), and 4(f)].

According to result 1, the Fourier spectrum of each of these gratings consists of two impulse rings (copies of the spectra of the original circular gratings of Subsection 6.A) that are shifted to the symmetric points $(u, v)$ $=(b f, c f)$ and $(u, v)=-(b f, c f)$. It is interesting to note that when the two shifted rings intersect each other (i.e., when $\sqrt{(b f)^{2}+(c f)^{2}}$ is smaller than the ring's ra- dius $f$, namely, $b^{2}+c^{2}<1$ ), the eccentric equispaced grating obtained in the image domain is elliptic [Fig. 4(e)]; when the two shifted rings are tangential (i.e., $b^{2}$ $+c^{2}=1$ ) the grating obtained in the image domain is parabolic [Fig. 4(d)], and when the two shifted rings are mutually exclusive (i.e., $b^{2}+c^{2}>1$ ), the grating is hyperbolic [Fig. 4(f)].

In a similar way, result 1 can be also used to obtain the spectra of eccentric zone gratings, eccentric sphere projection gratings, eccentric ellipsoid projection gratings [Fig. $4(\mathrm{~g})]$, etc.

\section{APPENDIX B: EFFECTS OF THE TRANSFORMATION $g(x, y)=g_{1}(x, y)+c$}

Let $g_{1}(x, y)$ be the bending transformation of the curvilinear gratings $r_{1}(x, y)=\cos \left[2 \pi f g_{1}(x, y)\right]$ and $r_{2}(x, y)$ $=\sin \left[2 \pi f g_{1}(x, y)\right]$. The addition of a constant $c$ to $g_{1}(x, y)$ is equivalent to a translation of the generating surface $z=g_{1}(x, y)$ by a constant $c$ along the $z$ axis. This affects in the image domain the phase of the gratings $r_{1}(x, y)$ and $r_{2}(x, y)$. However, the influence of such a phase change on the spectral domain is more complex:

Using the trigonometric identity $\cos (\alpha+\beta)$ $=\cos \alpha \cos \beta-\sin \alpha \sin \beta$, we obtain

$$
\begin{aligned}
r_{3}(x, y)= & \cos \left(2 \pi f\left[g_{1}(x, y)+c\right]\right)=r_{1}(x, y) \cos (2 \pi f c) \\
& -r_{2}(x, y) \sin (2 \pi f c) .
\end{aligned}
$$

Since $\cos (2 \pi f c)$ and $\sin (2 \pi f c)$ are constants, the spectrum of the grating $r_{3}(x, y)$ is

$$
R_{3}(u, v)=\cos (2 \pi f c) R_{1}(u, v)-\sin (2 \pi f c) R_{2}(u, v),
$$

namely, a weighted sum of the spectra $R_{1}(u, v)$ and $R_{2}(u, v)$, with the weighting coefficients $\cos (2 \pi f c)$ and $\sin (2 \pi f c)$.

Similarly, the spectrum of the grating $r_{4}(x, y)$ $=\sin \left\{2 \pi f\left[g_{1}(x, y)+c\right]\right\}$ is

$R_{4}(u, v)=\sin (2 \pi f c) R_{1}(u, v)+\cos (2 \pi f c) R_{2}(u, v)$.

\section{REFERENCES AND NOTES}

1. I. Amidror, "Fourier spectrum of radially periodic images," J. Opt. Soc. Am. A 14, 816-826 (1997).

2. A. V. Baez, "Fresnel zone plate for optical image formation using extreme ultraviolet and soft x radiation," J. Opt. Soc. Am. 51, 405-412 (1961).

3. T. R. Welberry and R. P. Williams, "On certain non-circular zone plates,” Opt. Acta 23, 237-244 (1976).

4. N. Abramson, "The holo-diagram. IV: a practical device for simulating fringe patterns in hologram interferometry," Appl. Opt. 10, 2155-2161 (1971).

5. J. Feldman, "Diffraction gratings," U.S. patent $3,628,849$ (1971); S. H. Macomber, "Curved grating surface emitting distributed feedback semiconductor laser," U.S. patent 5,345,466 (1994); T. Shiono, "Optical disk head including a light path having a thickness and width greater than the light beam wavelength by a predetermined, amount," U.S. patent 5,317,551 (1994).

6. R. N. Bracewell, The Fourier Transform and its Applications, 2nd ed. (McGraw-Hill, Reading, N.Y. 1986), p. 241. On alternative conventions used in literature and the relationships between them, see also pp. 7 and 17 .

7. Note that the term "curvature" is defined in mathematics in a different way; see, for example, R. Courant, Differential 
and Integral Calculus (Wiley-Interscience, New York, 1988), Vol. II, p. 86.

8. A. B. Ivanov, "Surface of the second order," in Encyclopaedia of Mathematics (Kluwer Academic, Dordrecht, The Netherlands, 1993), Vol. 9, p. 82.

9. I. N. Bronshtein and K. A. Semendyayev, Handbook of Mathematics (Springer-Verlag, Berlin, 1997), pp. 209-214.

10. O. E. Myers, Jr., "Studies of transmission zone plates," Am. J. Phys. 19, 359-365 (1951).

11. H. H. M. Chau, "Zone plates produced optically," Appl. Opt. 8, 1209-1211 (1969).

12. A. Erdélyi, (ed.), Tables of Integral Transforms (McGrawHill, New York, 1954), Vol. 1, p. 24.

13. R. N. Bracewell, Two Dimensional Imaging (Prentice-Hall, Englewood Cliffs, N.J., 1995), p. 166.

14. A. Erdélyi, (ed.), Tables of Integral Transforms (McGrawHill, New York, 1954), Vol. 1, p. 23.

15. R. N. Bracewell, The Fourier Transform and its Applications, 2nd ed. (McGraw-Hill, Reading, N.Y., 1986), p. 244.

16. The hyperbolic zone grating is also known as Girard grille; see, for example, S. Ananda Rao, "On the holographic simulation of a Girard grille," reprinted in Selected Papers on Zone Plates, SPIE Milestone Series, Vol. MS 128 (SPIE; Bellingham, Wash., 1996), pp. 386-387.
17. F. Oberhettinger, Tables of Bessel Transforms (SpringerVerlag, Berlin, 1972), p. 12. Note that in Eq. $2.57 y^{1 / 2}$ is erroneously missing in the right-hand side of the transform.

18. M. R. Spiegel, Mathematical Handbook of Formulas and Tables (McGraw-Hill, New York, 1968), pp. 136-139.

19. A. Erdélyi, (ed.), Tables of Integral Transforms (McGrawHill, New York, 1954), Vol. 1, p. 27.

20. R. N. Bracewell, Two Dimensional Imaging (Prentice-Hall, Englewood Cliffs, N.J., 1995), pp. 130-131.

21. A. Erdélyi, (ed.), Tables of Integral Transforms (McGrawHill, New York, 1954), Vol. II, p. 36.

22. A. Erdélyi, (ed.), Tables of Integral Transforms (McGrawHill, New York, 1954), Vol. II, p. 39.

23. F. Oberhettinger, Tables of Fourier Transforms and Fourier Transforms of Distributions (Springer-Verlag, Berlin, 1990), p. 27.

24. See, for example, in J. D. Gaskill, Linear Systems, Fourier Transforms, and Optics (Wiley, New York, 1978), pp. 308309; or in R. N. Bracewell, Two Dimensional Imaging (Prentice-Hall, Englewood Cliffs, N.J., 1995), pp. 159-161.

25. A. W. Lohmann and D. P. Paris, "Variable Fresnel zone pattern,” Appl. Opt. 6, 1567-1570 (1967). 